\title{
Femmes et féminité dans l'œuvre de Claude Simon : Recension critique
}

Alastair B. Duncan

\section{(2) OpenEdition}

1 Journals

Édition électronique

URL : http://journals.openedition.org/ccs/853

DOI : $10.4000 /$ ccs. 853

ISSN : 2558-782X

Éditeur :

Presses universitaires de Rennes, Association des lecteurs de Claude Simon

\section{Édition imprimée}

Date de publication : 31 mars 2013

Pagination : 25-36

ISBN : 9782354121785

ISSN : $1774-9425$

\section{Référence électronique}

Alastair B. Duncan, « Femmes et féminité dans l'œuvre de Claude Simon : Recension critique », Cahiers Claude Simon [En ligne], 8 | 2013, mis en ligne le 21 septembre 2017, consulté le 07 mai 2019. URL :

http://journals.openedition.org/ccs/853; DOI : 10.4000/ccs.853 


\title{
Femmes et féminité dans l'œuvre de Claude Simon : Recension critique
}

\author{
Alastair B. DUNCAN \\ Université de Stirling
}

Qu'a-t-on écrit sur les femmes et la féminité dans l'œuvre de Simon ? Le but de cette communication est de faire un rapide tour d'horizon des principaux axes de la critique. J'en distinguerai trois : des commentaires sur la représentation des femmes en tant que personnages; une approche qui situe la femme et les rapports entre homme et femme dans un contexte psychanalytique ; des commentaires qui, participant de plusieurs approches, mettent l'accent sur la mobilité de l'œuvre. En fin de parcours, je me permettrai quelques réflexions personnelles.

\section{La représentation des femmes}

Un certain nombre de critiques ont trouvé des traits récurrents dans la représentation des personnages de femmes dans l'œuvre de Simon, souvent en faisant la comparaison avec la représentation des hommes. "Dans son œuvre [...], dit Jean Duffy, la femme est 
perçue comme essentiellement autre ${ }^{1}$. " Même son de cloche chez Aline Baehler : "Dans La Route des Flandres, les femmes relèvent fondamentalement d'une autre espèce ${ }^{2}$ ". Christine Makward acquiesce et précise : "En général, Simon représente la femme comme une autre énigmatique, un partenaire érotique et un sujet ${ }^{3}$ ». Stéphanie Orace définit, d'une toute autre façon, la différence qu'elle perçoit entre la représentation des figures masculines et féminines : "les femmes en général chez Simon mobilisent les isotopies de la rigidité, la raideur, les hommes s'inscrivant au contraire du côté du mouvement, du voyage, du départ ${ }^{4}{ }$. Jacques Isoléry défend un autre point de vue : " [l]'univers simonien semble ainsi réparti en deux genres, chacun possédant bel et bien ses propres propriétés. Dureté du cru masculin contre flux hémorragique féminin ${ }^{5}$ ". L'analyse de Celia Britton comporte un jugement critique : "La représentation des personnages féminins dans les romans (à l'exception de Louise dans L'Herbe et peut-être de Batti dans Les Géorgiques) est partout négative et stéréotypée : les vieilles femmes sont soit terrifiantes (Histoire), soit ridicules (Sabine), soit d'une sainteté asexuelle (Marie), tandis que les jeunes sont exclusivement des objets sexuels et visuels ${ }^{6}$ ». John Fletcher propose une " typologie sommaire »

1. Jean Duffy, «M(i)sreading Claude Simon : a partial analysis ", Forum for Modern Language Studies, 27, no. 3, 1987, p. 228. Nous traduisons, comme toutes les citations en anglais qui suivent.

2. "L'Échange des femmes dans La Route des Flandres ", L'Écriture du féminin/ masculin, Ralph Sarkonak (dir.), La Revue des Lettres modernes, série Claude Simon, $\mathrm{n}^{\circ} 2,1997$, p. 21.

3. "Aspects of bisexuality in Claude Simon's Works ", dans Orion blinded, Randi Birn et Karen Gould (dir.), Lewisburg, Bucknell University Press, 1981, p. 219.

4. Stéphanie Orace, "Mouvement et immobilité de Claude Simon ", dans Claude Simon: Allées et venues. Actes du colloque international de Perpignan (14 et 15 mars 2003), Jean-Yves Laurichesse (dir.), Cahiers de l'Université de Perpignan, Presses Universitaires de Perpignan, ${ }^{\circ}$ 34, 2004, p. 134.

5. "Entre masculin et féminin : le schème de l'impassible ", Critique, n 584/585, janvier-février 1996, p. 51. Isoléry ajoute : "Mais ces principes ne sont tels et ne peuvent fonctionner l'un par rapport à l'autre que dans leur mutuelle complémentarité " .

6. Claude Simon: Writing the visible, Cambridge, Cambridge University Press, 1987, p. 167. 
des femmes chez Simon : "Il s'agit, d'une part, de la jeune fille raide, gauche et innocente [...] L'autre visage est celui de la femme voluptueuse, pleine d'expérience, dévorante et quelque peu putain, voire nymphomane ${ }^{7}$. Dans la première catégorie, Fletcher, se référant surtout aux premiers livres de Simon, range Belle dans Le Tricheur (1945), Véra dans La Corde raide (1947), Éliane dans Gulliver (1952), Edith dans Le Sacre du printemps (1954) et Cécile dans Le Vent (1957) - auxquelles il ajoute Marie dans L'Herbe (1958) : celle qui «n’a jamais tenu un homme embrassé, [...] n’a jamais enfanté ${ }^{8}$; ; dans la deuxième catégorie, il cite des femmes aperçues ou fantasmées, qui apparaissent dans Le Sacre du printemps (p. 15) ou La Bataille de Pharsale (1969) (OE, p. 622-623) - et évidemment Corinne de La Route des Flandres (1960).

A partir de ce premier échantillon, on constate que la critique s'est surtout intéressée aux jeunes femmes. L'évaluation négative que nous avons discernée chez Britton, se retrouve chez d'autres, surtout dans des articles qui adoptent plus ou moins explicitement une approche féministe. Duffy annonce cette approche dans le titre même de son article, titre à la fois franc et nuancé : « $M(i)$ sreading Claude Simon : a Partial Analysis" ». Les jugements de la critique sont sévères. Elle parle de " la réduction de la femme à la somme de ses parties sexuelles "; "du point de vue de la femme, dit-elle, L'Herbe est un roman défaitiste, ne proposant aucune alternative au sacrifice de soi du mariage bourgeois ${ }^{10} \%$. Dans le numéro spécial de L'Esprit créateur consacré à Simon en 1987, deux articles ont proposé des lectures féministes de La Route des Flandres. Pour Lynn Higgins, ce roman de guerre montre une solidarité entre hommes : c'est " un texte qui fait le récit du désir mâle en réalisant l'absence des femmes, et en produisant la femme en tant que texte, en pure représentation ${ }^{11} »$. Higgins interprète la révolte de Corinne et surtout

7. "Érotisme et création, ou la mort en sursis », Entretiens, n 31, Claude Simon, 1972, p. 132-133.

8. Fletcher, ibid., p. 133, citant L'Herbe, Minuit, p. 257.

9. Double jeu de mot : "Mal/Mme lire Claude Simon : une analyse partiale/ partielle ".

10. Duffy, art. cit., p. 230 et 233.

11. "Gender and war narrative in La Route des Flandres ", L'Esprit créateur, 27, 
sa fuite comme un moyen pour le romancier d'expulser le personnage féminin du roman : en quittant la chambre de l'hôtel où elle a couché avec Georges, elle laisse le champ libre aux fantasmes et à la parole des hommes. Winifred Woodhull renchérit en commentant l'infantilisation de Corinne ; comme Higgins, elle remarque que la femme est privée de parole et par conséquent exclue de l'Histoire avec un grand H. En outre, Woodhull critique les critiques de Simon, notamment Ricardou et Stephen Heath, en montrant comment, inconsciemment dans leur discours, ils universalisent l'expérience des hommes ${ }^{12}$. Dix ans plus tard, Woodhull revient à la charge. Son analyse des Géorgiques allie féminisme et post-colonialisme :

tout en signalant la force critique du roman de 1981 qui met en contact diverses identités qui sont à la fois produites et fissurées par une variété de discours culturels et historiques, j’avancerai que la référence plurielle du texte ne l'empêche pas de recycler quelques vieilles histoires, notamment celles de l'inévitable subordination des femmes et de la supériorité culturelle de l'Europe du Nord. ${ }^{13}$

Woodhull constate que ce roman largement consacré à l'époque révolutionnaire " anticipe le nouveau régime " où les femmes - elle cite la première femme de LSM et Batti - seront « exclues de la place publique de la démocratie ${ }^{14}$ ".

Le numéro spécial "Claude Simon " de L'Esprit créateur était sous la direction de Mária Minich Brewer. Deux ans plutôt, elle avait publié un article qui avance une tout autre interprétation du rôle de Corinne dans La Route des Flandres. Selon la thèse de Brewer, dans l'œuvre de Claude Simon " la référence à des formes canoniques de l'art [...] a pour but de présenter le texte en tableau vivant, masquant le processus narratif, l'écriture et le désordre des événements historiques qui contrediraient sa cohérence idéale et

$\mathrm{n}^{\circ}$ 4, hiver 1987, p. 19.

12. «Reading Claude Simon : Gender, ideology, representation ", L'Esprit créateur, 27, ibid., p. 5-16.

13. "Référents pluriels et vieilles histoires dans Les Géorgiques ", L'Écriture du féminin/masculin, op.cit., p. 78.

14. Art. cit., p. 82. 
intemporelle ${ }^{15}$ ". Dans La Route des Flandres, la féminité devient interchangeable avec cet " alibi esthétique » :

Dans les échanges en temps de guerre entre Georges et Blum, Corinne fournit une forme d'ancrage, de cohérence et d'intensité pour leurs récits ; elle est un prétexte libidinal, un simple figurant, un fantasme qui se désagrège quand, ayant écouté les histoires de guerre de Georges, elle proteste contre sa manipulation en tant qu'objet d'histoires de soldats [...] La construction de la femme en alibi ne s'écroule que quand elle refuse d'occuper la place qu'on lui assigne dans l'économie narrative ${ }^{16}$.

\section{Lapproche psychanalytique}

La critique de tendance psychanalytique, voire psychobiographique s'est fixé pour tâche de comprendre la représentation des femmes chez Simon. Le ton est donné par Britton : «La représentation des personnages féminins est si visiblement déterminée par une configuration d'angoisses et de désirs plus ou moins avoués que la question de leur attacher une objectivité quelconque ne se pose pas [...] ce qui est représenté n'est pas une femme mais $[\ldots]$ la construction de la sexualité mâle - non pas un personnage réaliste mais la projection des fantasmes du sujet ». Et elle ajoute : "Il est également pertinent que les protagonistes de Simon ne soient aucunement des héros sexuels triomphants les sentiments dominants sont l'échec, la peur, la désespérance et surtout la perte ${ }^{17}$ ». Dällenbach a posé une première pierre pour expliquer cette configuration de sentiments : « le roman simonien, dit-il, essentiellement matrilinéaire, est bâti autour d'un vide peuplé par une absence essentielle - celle du Père ${ }^{18} »$. En se basant sur Freud et, en partie, sur Lacan, Dällenbach envisage surtout ce qui prend la place de cette absence, c'est-à-dire le roman familial : la recherche

15. "Claude Simon : the critical properties of painting ", The Review of Contemporary Fiction, vol. 5, 1985, numéro spécial Claude Simon, p. 105.

16. Art. cit., p. 106.

17. Celia Britton, Claude Simon, Writing the Visible, Cambridge, Cambridge University Press, 1987, p. 167.

18. Claude Simon, Seuil, 1982, p. 91. 
identitaire qui se poursuit à travers la création de pères substituts et la multiplication de figures de mère - et les rapports troubles qu'entretient le fils avec ses figures de père et de mère. Dans les figures de mère, Dällenbach voit apparaître parfois « le fantasme archaïque entre tous (et par conséquent dont il est le plus difficile de se libérer au dire de la psychanalyse) de la mère phallique ${ }^{19}$ ". Anthony Cheal Pugh, parlant de la mère dans Histoire, explique : " avec la mort du père, la mère est devenue la figure qui possède le pouvoir symbolique de châtrer, et de punir les impulsions incestueuses ${ }^{20}$ " : son visage en " lame de couteau » - image récurrente - la rend aussi terrifiante que pitoyable.

Alastair Duncan a commenté un drame odipien dans La Route des Flandres ${ }^{21}$. De Reixach est un père substitut. Par le brassage des images, des figures féminines du roman - Corinne, la paysanne aperçue dans la grange, la jeune femme de l'imbroglio paysan - se fondent en " une addition de toutes les femmes, vieilles ou jeunes" (OE, p. 295). Corinne, la jeune femme fantasmée par des adolescents sevrés de femme, devient aussi une figure de mère. En couchant avec la veuve de son cousin éloigné, Georges prend la place du père et réalise le fantasme d'Edipe - ce qui ne sert qu'à renforcer sa crise identitaire. Pascal Mougin a suivi l'évolution du traitement de la figure de la mère à travers les romans. Selon lui, les traits de la mère sont constants ; elle est associée à un déni de la mort ; son souvenir est « une force agissante dans le parcours du fils ${ }^{22}$ ». Dans Le Tricheur

19. Op. cit., p. 93.

20. Anthony Cheal Pugh, Simon 'Histoire', Londres, Grant and Cutler, 1982, p. 53.

21. Claude Simon, Euvres, Gallimard, coll. «Bibliothèque de la Pléiade », p. 13021304. Voir aussi Claude Simon. Adventures in Words, Manchester, Manchester University Press, 1994, nouvelle édition revue et augmentée, 2002, p. 112-118, et "Allées et venues familales chez Claude Simon", dans Claude Simon: Allées et venues, op. cit., p. 171-181.

22. "La mère, la mère toujours recommencée ", dans Claude Simon: Allées et venues, ibid., p. 184. Aux premières pages de cet article (p. 183-185), Mougin résume l'argument de ses articles précédents et présente l'argument qu'il va développer sur Le Tramway. Pour plus de détail, voir Mougin, "La Femme, L'Histoire et le guerrier : transformations d'un imaginaire de La Route des Flandres à L'Acacia ", dans L'Écriture du féminin/masculin, op.cit., p. 99-123 ; Lecture de 
(1945) et Histoire (1967), la mère morte serait la source d'une obsession préœedipienne qui empêche le passage à l'âge d'homme. Une comparaison entre La Route des Flandres de 1960 et L'Acacia de 1989 fait apparaitre un certain apaisement : " la femme cesse d'être le centre obsédant d'un fantasme originel et eschatologique du guerrier ${ }^{23}$ ». Le Tramway (2001) dépasse cette étape. Dans deux images de la mère, « l'écart entre la mourante et la mère aimante est affirmé24 ». S'appuyant sur Denil et mélancolie de Freud, Mougin voit dans ce roman le travail de deuil accompli ${ }^{25}$.

\section{La mobilité à l'œuvre}

Dans un autre article paru dans L'Esprit créateur, Aline Baehler remarque que la typologie des femmes dans l'œuvre de Simon est complexe. Les « femmes rouges " - groupe qui correspond à peu près à la deuxième catégorie de Fletcher - forment un ensemble aux traits communs ; mais ces traits réapparaissent chez d'autre femmes - les femmes " pêche ", dont les traits distinctifs se trouvent à leur tour chez celles du « clan des Cerises » : « le brouillage des référents provoque donc un amalgame des figures ${ }^{26} »$. Stéphanie Orace a repris l'idée de cette typologie glissante. Elle remarque que des motifs associés aux "femmes rouges »- maquillage outré, peignoir à fleurs, la couleur rouge des accessoires - se retrouvent en partie chez d'autres femmes :

'L'Acacia' de Claude Simon. L'imaginaire biographique, Minard, 1997, Archives des lettres modernes, $\mathrm{n}^{\circ} 267$, p. 49-67 et 110-112; « Du Tricheur au Jardin des Plantes: la figure de la mère défunte ", dans Le Jardin des Plantes de Claude Simon, Actes du colloque de Perpignan [27 mars 1999], Jean-Yves Laurichesse (dir.), Cahiers de l'Université de Perpignan, $\mathrm{n}^{\circ}, 30,2000$, p. 87-99 ; « Histoire: L'Aventure espagnole comme quête oedipienne ", dans Lectures de Histoire, Ralph Sarkonak (dir.), La Revue des Lettres modernes, série Claude Simon, n³ 3, 2000, p. 125-141.

23. Lecture de 'L'Acacia' de Claude Simon. Limaginaire biographique, Minard, 1997, p. 110.

24. P. Mougin, «La mère, la mère toujours recommencée ", p. 187.

25. Pour une perspective plus sombre sur Le Tramway, voir Sjef Houppermanns, "L'Homme Couché et l'Homme Debout ", dans Claude Simon: Allées et venues, op. cit., p. 62.

26. "Aspects du personnage simonien : Corinne ", L'Esprit Créateur, 2, n 4 , hiver 1987 , p. 31. 
" la femme-putain, outrageusement maquillée, dont Corinne et Sabine sont les symboles, croise la femme aimée que sont Rose ou Hélène ${ }^{27}$ ". Christine Makward a fait s'étendre ces glissements au-delà des figures de femmes. Elle remarque trois tendances qui brouillent la distinction entre féminité et masculinité : « la question de l'homosexualité ; des cas de 'transsexualisme' psychologique, là où un personnage tend à 'transgresser' son rôle sexuel tel qu'il est défini par la culture ; et enfin, l'attention 'féminine' consacrée au corps et la diffusion de l'érotisme dans le texte entier dans les dernières œuvres $^{28}$ ". Les " dernières œuvres " au moment où Makward publie cet article sont celles des années soixante-dix, Les Corps conducteurs (1971), Triptyque (1973) et Leçon de choses (1976) ; les personnages transgressifs sont Montès - bisexuel ou "sans sexe ", selon Makward - et Louise :

la restructuration de l'identité psycho-sexuelle de Louise consiste en un déplacement de l'investissement libidinal, passant de l'archä̈que - la gratification érotique et la 'libération' de la famille - au symbolique : en apparence, Louise se conformera à la loi patriarcale tandis qu'en fait elle $s^{\prime}$ identifie au triomphe silencieux sur le temps de $\mathrm{Marie}^{29}$.

La mobilité des traits chez Simon va encore plus loin. Stéphanie Orace l'avait remarqué en inscrivant les glissements de la féminité dans le contexte des multiples déplacements de l'œuvre, thématiques, fictionnels, formels et stylistiques. Mary M. Perramond y souscrit également. Elle se base sur l'étude d'objets dans Les Géorgiques tels que le camée et le buste ; à ses yeux, " ce couple de têtes tranchées aide à brouiller les identités des différents personnages [...] et même [...] à abolir toute opposition binaire ${ }^{30}$ ". Aline Baehlin abonde dans le même sens. Revenant sur la question de l'échange des femmes

27. «Mouvement et immobilité de Claude Simon", dans Claude Simon: Allées et venues, op. cit., p. 150.

28. "Aspects of bisexuality in Claude Simon's Works ", dans Orion Blinded, Randi Birn et Karen Gould (dir.), Lewisburg, Bucknell University Press, 1981, p. 220-221.

29. Ibid., p. 225.

30. "L'indistinction de l'autre et le discours du désir dans Les Géorgiques", L'Écriture du féminin/masculin, op.cit., p. 61. 
dans La Route des Flandres, elle abandonne en fin de parcours sa perspective lévi-straussienne et les distinctions entre hommes et femmes, pour commenter " le plaisir que nous procure le texte simonien " :

Je veux parler de la subversive fécondité d'une écriture hautement incestueuse $[\ldots]$ qui ravit parce qu'elle transforme le langage, source de toutes nos divisions, en une fabuleuse mise en rapport des sexes, en une formidable copulation des mots qui rassemblent ce qui est épars jusqu'à ce que, fantasmatiquement dépassée, s'abroge toute notion de désaccord ${ }^{31}$.

Quelle serait donc la pertinence des études qui se penchent justement sur les différences entre hommes et femmes, entre masculinité et féminité ? Dans son article de 1987, Woodhull s'était déjà posé la question : "Dans un contexte où [...] les frontières entre Georges, Corinne, etc, le cheval et la terre sont remarquablement fluides, quel sens y a-t-il à parler de la distinction des genres ?32 ". La réponse de Woodhull ne fait pas de doute, parce que, nous l'avons vu, elle est remontée au créneau dans son article de 1997. D'autres, parmi ceux qui sont restés au plus près du texte, donnent une réponse plus nuancée. C'est le cas de Mireille Calle-Gruber qui analyse un passage de La Route des Flandres (Minuit, p. 82-83). Elle y trouve " une intersection des champs du féminin et du masculin ${ }^{33}$ ". Elle conclut que les différences sexuelles "sont indissociables de l'ensemble constellaire de toutes les différences, où elle s'éclairent réciproquement et tour à tour ${ }^{34}$ ". Raymond Jean, dans son analyse remarquable de deux passages similaires et contrastés - La Route des Flandres, (Minuit, p.257-292), et La Bataille de Pharsale, (Minuit, p.211-247) - commente la spécificité de l'écriture de Simon :

31. "L'échange des femmes dans La Route des Flandres ", L'Écriture du féminin/ masculin, ibid., p. 32-33.

32. «Reading Claude Simon : Gender, ideology, representation ", L'Esprit créateur, 27, op.cit., p. 12.

33. "Tourner autour ", Littérature, n 142, juin 2006, La différence sexuelle en tous genres, p. 98.

34. Ibid., p. 101. 
Nous sommes très loin de tout ce qui peut lier le désir à une transgression (Bataille) ou à une activité pure du regard (RobbeGrillet) [...] L'amour, chez lui [Simon], loin de supposer éloignement ou recul, implique le contact et la fusion des corps, met en jeu une totalité sensorielle vécue et décrite dans la " participation ", se situe volontiers à un niveau que l'on pourrait appeler "vitaliste " et même « nutritionnel ».

D'où une richesse d'écriture exceptionnelle, dont l'un des éléments essentiels est la présence d'un réseau connotatif, associatif et métaphorique très ramifié et d'une singulière abondance, qui tend à rendre sensible, à inscrire littéralement dans le texte cette réalité organique des choses du sexe, en la nourrissant d'images et de formes empruntées surtout au monde végétal et animal ${ }^{35}$.

Perramond développe cette idée en montrant comment le réseau connotatif sexuel parcourt en filigrane bien des épisodes des Géorgiques a priori non sexuels par exemple les premières visites du garçon au cinéma ${ }^{36}$. Ralph Sarkonak, en introduisant la livraison de La Revue des Lettres modernes consacrée au féminin/masculin se demande même s'il ne faudrait pas " envisager la possibilité que le jouissif simonien atteigne son plein ou son trop-plein synergique dans les passages à première vue non-sexuels à cause des nombreuses allusions suggestives qui s'y trouvent ${ }^{37}$ ».

\section{Pistes d'étude}

Que reste-t-il à approfondir ? On peut penser que la question des différences entre les genres n'est pas à évacuer : l'œuvre de Simon comporte des personnages, même si leur statut est problématique et leurs contours sont flous. Surtout dans les premiers romans, les contrastes entre hommes et femmes sont patents. L'homme pense, cherche à voir un ordre dans le chaos du monde ou à faire la révolution : Louis dans Le Tricheur, Gérard Faure dans Gulliver, Bernard et son beau-père dans Le Sacre du printemps. Chacun, à sa façon, fait son apprentissage de vie, doit s'avérer battu, cherche

35. "Les signes de l'éros », Entretiens, n 31, Claude Simon, 1972, p. 121-122. 36. Art. cit., p. 59-60.

37. « De l'érotique à l'informatique ", L'Écriture du féminin/masculin, op. cit., p. 4. 
l'oubli, parfois la petite mort dans les bras de la femme. La femme, en revanche, ne pense pas. Elle est près de la terre. « Les femmes sont plus animales que nous, dit Louis dans Le Tricheur, et elles vivent plus avec leurs $\operatorname{corps}^{38}$ ". On pense à la mère de Belle, à Josie dans Le Sacre du printemps, et, malgré tout ce qui la distingue, à Marie dans L'Herbe. Toutes ces femmes, je cite Le Tricheur, « acceptent la succession des sensations, sans chercher à leur découvrir un lien ${ }^{39}$ ". Et pourtant, déjà dans ces romans et ceux qui les suivent, des traits associés aux hommes apparaissent chez des femmes, et vice versa. Les frères de Chavannes dans Gulliver, le capitaine Ceccaldi dans Le Sacre du printemps sont doués d'une sorte d'intégrité primitive. Le grand-père analphabète dans L'Herbe, les soldats-paysans dans La Route des Flandres sont aussi près de la terre que les femmes. D'autre part, certaines femmes sont lucides et tourmentées : Éliane dans Gulliver, Edith dans Le Sacre du printemps, Cécile dans Le Vent. Le plus remarquable de ces personnages est, bien sûr, Louise dans L'Herbe qui subit une initiation tout à fait comparable aux hommes, y compris la recherche de l'oubli dans l'étreinte d'un corps.

Il faudra aussi revenir sur les femmes mûres et âgées. Là encore, il y a des figures récurrentes : la vieille femme, symbole de mort et de castration, « horrible et goyesque vieille » de La Route des Flandres ${ }^{40}$, tueuse de lapins dans Triptyque. Cette figure est sans doute apparentée à la domestique, veilleuse de mourante dans L'Herbe et Le Tramway. Nathalie Piégay-Gros a cerné la spécificité de la domestique chez Simon :

Si la bonne, dans les romans de Claude Simon, est donc une figure de la soumission et du dévouement conforme aux représentations et à l'imaginaire de la société bourgeoise et du roman réaliste et naturaliste du XIX ${ }^{e}$ siècle, il l'infléchit de deux manières : d'une part en laïcisant radicalement ce dévouement ; d'autre part en substituant à l'analyse en termes de pouvoir et de domination, ou de lutte des classes, une vision archaïque, quasi mythologique de la domestique ${ }^{41}$.

38. Sagittaire, 1945, p. 27.

39. Ibid, p. 27.

40. Claude Simon, E, p. 380.

41. «La figure de la domestique chez Claude Simon", dans Claude Simon: situations, Paul Dirkx et Pascal Mougin (dir.), ENS éditions, 2011, p. 137. 
Cette étude ouvre la voie à d'autres réflexions possibles sur le jeu avec les stéréotypes dans l'œuvre de Simon. Exemple le plus clair : le séducteur dans Leçon de choses: aucune trace chez lui des sentiments répertoriés par Celia Britton : "l'échec, la peur, la désespérance et surtout la perte ». Et que dire de la Corinne vieillissante que nous retrouvons dans Triptyque après l'avoir connue dans La Route des Flandres et dans Histoire : parodie de la pratique du retour des personnages à la Balzac, ou source d'une dimension affective que l'on n'associe guère avec ce roman?

Enfin, il faudrait placer féminité et masculinité dans le contexte du " tout au même plan », ce principe qui domine l'esthétique de Simon pendant une décennie à partir de La Bataille de Pharsale. Y a-til une tension entre, d'une part, la volonté d'échapper à la tradition judéo-chrétienne d'une sexualité dominée par les sentiments de transgression et de culpabilité - d'où les descriptions ultra - précises, objectives du coït dans Triptyque - et, d'autre part, se dégageant de Triptyque aussi bien que de Histoire, le sentiment que la sexualité est une force irrésistible apportant malheur aux hommes et aux femmes? 